\title{
Der Zoom-Fatigue entgegenwirken
}

Von der sog. Zoom-Fatigue ist beinahe jeder Schüler, Student und Arbeitnehmer/-geber betroffen. Unter dem Begriff ist eine anhaltende Erschöpfung und Müdigkeit zu verstehen, die durch die Teilnahme an Video-Konferenzen verursacht wird. Zurückzuführen ist das Phänomen auf monotone (Hoch-)Schul-/Arbeitsalltage, wenig Bewegung, andauerndes Blicken auf einen Bildschirm während Videokonferenzen sowie des daraus resultierenden erhöhten Konzentrationsaufwands, um Sprache und Bild zu verstehen und $\mathrm{zu}$ interpretieren. Fachkreise gehen davon aus, dass auch nach der Covid-19-Pandemie keine Rückkehr zum vorherigen Arbeitsalltag eintreten wird, sodass digitale Besprechungen das Arbeitsleben nachhaltig prägen werden. Daher ist es wichtig, der Zoom-Fatigue gezielt entgegen $\mathrm{zu}$ wirken, um negative Folgen $\mathrm{zu}$ verringern oder sogar zu vermeiden. Hierzu können folgende Tipps beachtet werden:

\section{Bauen Sie Bewegung in Ihren Arbeitsalltag mit ein}

Im Home-0ffice bewegt man sich weniger als am eigentlichen Arbeitsplatz. Versuchen Sie in Ihrer Mittagspause einen Spaziergang einzulegen oder zwischenzeitlich im Stehen zu arbeiten, um Ihren Kreislauf zu unterstützen. Häufiges Lüften und viel Tageslicht helfen Ihnen ebenfalls dabei, sich besser konzentrieren zu können.

\section{Nutzen Sie adäquates Equipment}

Benutzen Sie Head-Sets und externe Bildschirme, um möglichen Ton- und Sichtproblemen sowie hohem Kon- zentrationsaufwand entgegenzuwirken. Darüber hinaus sollten Sie Ihre Internetverbindung überprüfen und evaluieren, ob der bisherige Netzanbieter Ihren Anforderungen gerecht wird.

3. Erleichtern Sie sich die Teilnahme an Videokonferenzen

Richten Sie Ihren Blick während digitaler Konferenzen für einige Sekunden auf etwas anderes, um Ihre Augen zu entlasten. Stellen Sie außerdem die Sprecheransicht für die Konferenzen an, wenn dies in Ihrem Programm möglich ist. Das ermöglicht Ihnen eine leichtere Interpretation des Gesagten, da Sie auch non-verbale Sprachelemente besser interpretieren können.

4. Gestalten Sie Ihre Pausen und Freizeit abwechslungsreich

Trotz der Pandemie gibt es viele Möglichkeiten, um Körper und Geist zu entlasten. Essen Sie bspw. nicht vor dem Laptop während Ihrer Pausen, sondern suchen Sie sich zur Entspannung einen anderen Platz. Ihre Freizeit sollten Sie gänzlich anders als Ihren Arbeitsalltag gestalten. Verbringen Sie daher Ihre Freizeit im Freien und gehen Sie bspw. Ihren (sportlichen) Hobbys nach oder legen Sie einen langen Spaziergang mit Ihrem Partner ein.

Christopher Maasz, M.Sc., Marburg 